\title{
Second-order statistics of non-Gaussian fluctuations of coherent waves reflected from disordered media
}

\author{
M. Nieto-Vesperinas \\ Instituto de Ciencia de Materiales, Consejo Superior de Investigaciones Científicas and Departamento de Física \\ de la Materia Condensada, Facultad de Ciencias, Universidad Autónoma, Cantoblanco, Madrid 28049, Spain \\ J. A. Sánchez-Gil \\ Instituto de Optica, Consejo Superior de Investigaciones Científicas, Serrano 121, Madrid 28006, Spain
}

(Received 19 January 1993; revised manuscript received 5 April 1993)

\begin{abstract}
We show that the second-order statistics of speckle patterns of scalar waves, multiply scattered on reflection by disordered media, may be non-Gaussian, even though the first-order statistics are Gaussian. This occurs at intermediate sizes of the illuminated samples of the medium that are large enough to produce Gaussian first-order statistics of the scattered field but are small enough to break down the socalled factorization approximation in directions of the incident and scattered wave vectors at which there are enhanced long-range angular-correlation peaks. We propose joint probability distributions of the scattered intensity that are given in terms of modified Bessel functions of the second kind.
\end{abstract}

Coherent effects associated with the multiple scattering of classical waves in dense media ${ }^{1-6}$ and by rough surfaces $^{7-10}$ have been a subject of great interest in the last few years. ${ }^{11-13}$ An important property of the speckle fluctuations is that they generally follow Gaussian firstorder statistics; namely, the real and imaginary parts of the complex amplitude are Gaussian-distributed and uncorrelated, its modulus has a Rayleigh probability density function (PDF), and its intensity has a negative exponential PDF. Conversely, it has been shown ${ }^{14,15}$ that strong disorder may cause this statistic to be non-Gaussian. Also, small samples (namely, illuminated areas of the medium with sides of a few wavelengths) have been shown to produce, both in multiple scattering ${ }^{15}$ and in single scattering, ${ }^{16,17}$ speckle fluctuations with nonGaussian first-order statistics.

In this paper we address some properties connected to the existence of long-range intensity correlations of the second-order statistics of the speckle fluctuations of reflected waves. These correlations have been hypothesized and observed ${ }^{18-22}$ for small illuminated samples of the medium, both in reflection and transmission; in addition, the effect on them of time-reversal symmetry has also been studied. ${ }^{23,24}$ Recently, we predicted enhanced long-range angular-correlation (ELRC) peaks in scattering from rough surfaces. ${ }^{25}$ These functions describe the degree of correlation between the successive speckle patterns of the scattered wave, which result as the direction of the incident wave vector varies. For small samples that produce multiple scattering, due to both this small size of the sample and the crossing of scattering paths, there is a low number of independent scatterers, hence these correlations can be interpreted in terms of the failure of the so-called factorization approximation and of the time-reversal symmetry that holds for multiple scattering paths on reflection (see Ref. 25 for more details). These peaks possess the very interesting property of being produced by the interference of multiple scattering paths and their time-reversed counterparts, thus consitituting an enhanced backscattering generalization to higher statistical moments of the intensity (cf. Refs. 8-10, 25, and 26).

Specifically, we shall show that there are sizes of the illuminated samples of the random medium such that even if they are sufficiently large to produce Gaussian firstorder statistics for the scattered field, these samples may still be small enough so that their second- and higherorder statistics are non-normal. We also state that this is what breaks down the factorization approximation, thus giving rise to the ELRC peaks.

We shall consider the speckle pattern at a direction of observation characterized by the wave vector $\mathbf{k}_{f}$ produced by scattering of a plane wave with wave vector $\mathbf{k}_{i}$, incident on the random medium. We shall then address another speckle pattern in the scattering direction $\mathbf{k}_{f}^{\prime}$ due to reflection of a second plane-wave incident with wave vector $\mathbf{k}_{i}^{\prime}$.

It was shown in Ref. 25 that the intensity angularcorrelation function (IAC)

$$
C\left(\mathbf{k}_{i}, \mathbf{k}_{f} ; \mathbf{k}_{i}^{\prime}, \mathbf{k}_{f}^{\prime}\right)=\frac{\left\langle\delta I\left(\mathbf{k}_{i}, \mathbf{k}_{f}\right) \delta I\left(\mathbf{k}_{i}^{\prime}, \mathbf{k}_{f}^{\prime}\right)\right\rangle}{\left\langle I\left(\mathbf{k}_{i}, \mathbf{k}_{f}\right)\right\rangle\left\langle I\left(\mathbf{k}_{i}^{\prime}, \mathbf{k}_{f}^{\prime}\right)\right\rangle},
$$

of the waves reflected from small samples of the random medium has two ELRC peaks, respectively, at

$$
\mathbf{k}_{i}+\mathbf{k}_{f}= \pm\left(\mathbf{k}_{i}^{\prime}+\mathbf{k}_{f}^{\prime}\right) \text {. }
$$

In Eq. (1), $\delta I=I-\langle I\rangle$ and $\langle\cdots\rangle$ denotes ensemble average.

We shall next show that the joint probability density function (JPDF)

$$
P\left(I\left(\mathbf{k}_{i}, \mathbf{k}_{f}\right), I\left(\mathbf{k}_{i}^{\prime}, \mathbf{k}_{f}^{\prime}\right)\right)
$$


is not jointly Gaussian for values of the wave vectors near those satisfying Eq. (2). This is the origin of the failure of the factorization approximation in these regions of the $\mathbf{k}$ vectors and, hence, of the appearance of the ELRC peaks.

We have made a numerical simulation of the multiple scattering of an $s$ polarized electromagnetic wave from a one-dimensional random rough surface, of profile $z=D(x)$, separating the vacuum from a perfect conductor (hence, the surface being constant in the $y$ direction). The plane of incidence is the $O X Z$ plane. The lack of a third-dimension dependence is made in order to save computation time, and since no depolarization effects will be accounted for, it is not relevant. Also, the assumption of a hard wall, or perfect conductor, renders a much less difficult (numerically) problem than a penetrable medium. ${ }^{25}$

Using the Monte Carlo procedure reported in Refs. 9 and 10, we generate random profiles with Gaussian statistics, zero mean, rms height $\sigma=1.9 \lambda$, and a Gaussian correlation function of the random heights with correlation distance $T=3.16 \lambda$. Each realization of the illuminated surface contains 300 sampling points and has a length $L=31.6 \lambda$. This involves ten sampling points per wavelength which is known to be accurate enough. ${ }^{9,26}$ Averages are made over 4000 realizations. In order to get smoother results, we choose Gaussian beams of width $W=L \cos \theta_{0} / 4$, propagating in the $\mathbf{k}_{i}$ and $\mathbf{k}_{i}^{\prime}$ directions, at angles $\theta_{0}$ and $\theta_{0}^{\prime}$ with the $O Z$ axis, respectively. We shall denote by $\theta$ and $\theta^{\prime}$ the scattering angles of $\mathbf{k}_{f}$ and $\mathbf{k}_{f}^{\prime}$ with the $O Z$ axis, respectively. In our notation, $\theta=-\theta_{0}$ and $\theta^{\prime}=-\theta_{0}^{\prime}$ represent backscattering directions. It should be emphasized that the first-order PDF of the intensity scattered from these surface samples is a negative exponential.

Figure 1 shows the JPDF: $P\left(I\left(0^{0}, 0^{0}\right) ; I\left(5^{0},-5^{0}\right)\right)$. At these values of the arguments, which satisfy Eq. (2), the IAC is enhanced and has a value approximately equal to 0.3. As $I$ and $I^{\prime}$ approach zero, this function has values larger than those corresponding to a negative exponential.

To better visualize the variation of the JPDF, we also

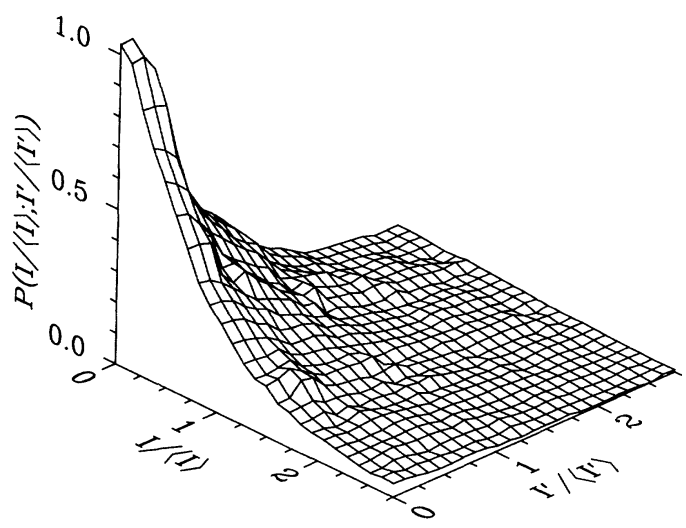

FIG. 1. Joint probability density function $P(I /\langle I\rangle$; $I^{\prime}\left\langle\left\langle I^{\prime}\right\rangle\right), I=I\left(0^{\circ}, 0^{\circ}\right)$, and $I^{\prime}=I\left(5^{0},-5^{0}\right)$, for reflection from a surface with $T=3.16 \lambda$ and $\sigma=1.9 \lambda$. show the sections of two of these functions by the planes $I\left(\theta_{0}, \theta\right)=0$ and $I\left(\theta_{0}, \theta\right)=I\left(\theta_{0}^{\prime}, \theta^{\prime}\right)$. Figure 2 shows $P\left(I\left(\theta_{0},-\theta_{0}\right) ; I\left(\theta_{0}^{\prime}-\theta_{0}^{\prime}\right)\right)$, for $I\left(\theta_{0},-\theta_{0}\right)=0.06, \theta_{0}=0^{0}$, and $\theta_{0}^{\prime}=3^{0}$ and $5^{\circ}$, respectively. On the other hand, in Fig. 3 the section: $P\left(I\left(\theta_{0},-\theta_{0}\right) ; I\left(\theta_{0},-\theta_{0}\right)\right)$ for $\theta_{0}=3^{0}$ and $5^{0}$, respectively, is displayed. As seen in these figures, for small values of the arguments, the computed JPDF has higher values than that corresponding to Gaussian statistics. The physical meaning of the JPDF given by Figs. 2 and 3 is a sparse speckle, namely, narrow sharp peaks and broad dark areas.

In order to give the law followed by these functions, we have made use of the $K$ distributions. ${ }^{27}$ These probability densities, which have been employed to describe the first-order statistics of non-Gaussian speckle patterns, ${ }^{17}$ depend on the modified Bessel functions of the second kind, $K_{n-1}$. They were introduced to characterize these spiky speckle fluctuations within the framework of a random walk with a finite number of steps, and read

$$
P_{n}(I)=\frac{2}{\Gamma(n)} \sqrt{n^{n+1}(I /\langle I\rangle)^{n-1}} K_{n-1}[2 \sqrt{n(I /\langle I\rangle)}] .
$$

In Eq. (3), $n \propto N, N$ being the number of independent scatterers.

We propose a two-dimensional generalization of these functions as the JPDF of the scattered intensity. Namely, this distribution is given by
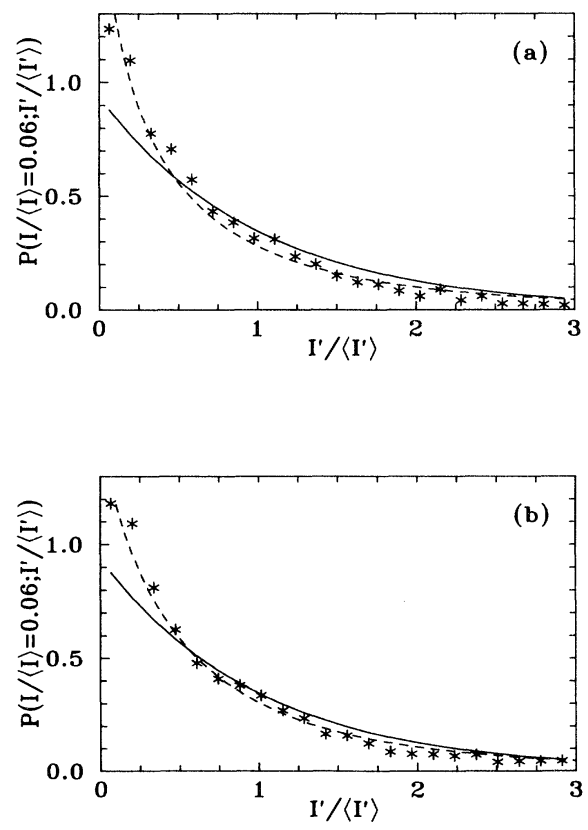

FIG. 2. (a) Section $P\left(I /\langle I\rangle=0.06 ; I^{\prime} /\left\langle I^{\prime}\right\rangle\right), I=I\left(0^{\circ}, 0^{\circ}\right)$, and $I^{\prime}=I\left(3^{0},-3^{0}\right) . T=3.16 \lambda$ and $\sigma=1.9 \lambda$. Asterisks: numerical calculation. Broken line: JPDF given by Eq. (4) with $n=2$. Full line: JPDF corresponding to Gaussian statistics [Eq. (9)]. (b) Same as (a) for $I^{\prime}=I\left(5^{0},-5^{0}\right), n=3$. 

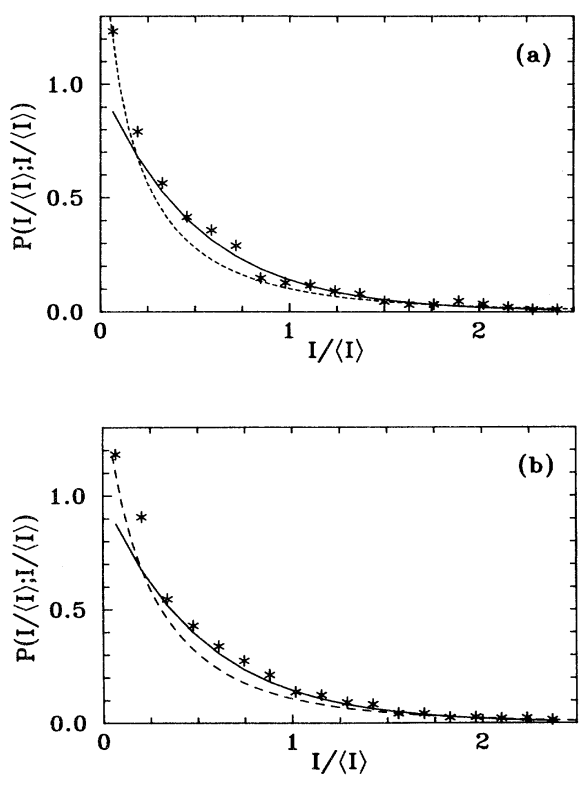

FIG. 3. (a) Section $P\left(I /\langle I\rangle ; I^{\prime} /\left\langle I^{\prime}\right\rangle=I\langle I\rangle\right), I=I\left(0^{0}, 0^{\circ}\right)$, and $I^{\prime}=I\left(3^{0},-3^{\circ}\right) . T=3.16 \lambda$ and $\sigma=1.9 \lambda$. Asterisks: numerical calculation. Broken line: JPDF given by Eq. (4) with $n=2$. Full line: JPDF corresponding to Gaussian statistics [Eq. (9)]. (b) Same as (a) for $I^{\prime}=I\left(5^{\circ},-5^{\circ}\right), n=3$.

$$
\begin{aligned}
P_{n}\left(I, I^{\prime}\right)= & \frac{2}{\Gamma(N)} \frac{\sqrt{n^{n+1} s^{n-1}}}{\langle I\rangle^{2}\left(1-|\mu|^{2}\right)} \\
& \times K_{n-1}(2 \sqrt{n s}) I_{0}\left(\frac{2 \sqrt{I I}|\mu|}{\langle I\rangle\left(1-|\mu|^{2}\right)}\right] .
\end{aligned}
$$

In Eq. (4), $I_{0}$ is the modified Bessel function of the first kind and zero order, $I=I\left(\mathbf{k}_{i}, \mathbf{k}_{f}\right)$, and $I^{\prime}=I\left(\mathbf{k}_{i}^{\prime}, \mathbf{k}_{f}^{\prime}\right)$. $\langle I\rangle=\left\langle I^{\prime}\right\rangle$. Also, $s$ stands for

$$
s=\frac{I+I^{\prime}}{\langle I\rangle\left(1-|\mu|^{2}\right)},
$$

$\mu$ being the angular complex coherence factor for the complex amplitude $A\left(\mathbf{k}_{i}, \mathbf{k}_{f}\right)$ of the scattered field. Namely,

$$
\mu\left(\mathbf{k}_{i}, \mathbf{k}_{f} ; \mathbf{k}_{i}^{\prime}, \mathbf{k}_{f}^{\prime}\right)=\frac{\left\langle A^{*}\left(\mathbf{k}_{i}, \mathbf{k}_{f}\right) A\left(\mathbf{k}_{i}^{\prime}, \mathbf{k}_{f}^{\prime}\right)\right\rangle}{\left(\left\langle I\left(\mathbf{k}_{i}, \mathbf{k}_{f}\right)\right\rangle\left\langle I\left(\mathbf{k}_{i}^{\prime}, \mathbf{k}_{f}^{\prime}\right)\right\rangle\right)^{1 / 2}}
$$

The index $n$ should increase both with $W$ (which limits the number of independent scatterers) and with the inverse of the magnitude of the ELRC peaks. Since, however, these peaks are due to the interference between terms of the form

$$
\left|A_{l m}\right|^{2} \exp \left[i\left(\mathbf{k}_{i}-\mathbf{k}_{i}^{\prime}\right) \cdot \mathbf{r}_{l}\right] \exp \left[-i\left(\mathbf{k}_{f}-\mathbf{k}_{f}^{\prime}\right) \cdot \mathbf{r}_{m}\right]
$$

and their time-reversed counterparts, and also of terms

$$
\left|A_{l m}\right|^{2} \exp \left[i\left(\mathbf{k}_{i}+\mathbf{k}_{f}^{\prime}\right) \cdot \mathbf{r}_{l}\right] \exp \left[-i\left(\mathbf{k}_{i}^{\prime}+\mathbf{k}_{f}\right) \cdot \mathbf{r}_{m}\right]
$$

and their time-reversed counterparts, then $n$ should increase with the inverse of the degree of coherence $v$ of these terms. In a continuous approach, this degree of coherence may be expressed as [cf. Eq. (6) of Ref. 25]

$$
v\left(\mathbf{k}_{i}, \mathbf{k}_{f} ; \mathbf{k}_{i}^{\prime}, \mathbf{k}_{f}^{\prime}\right)=\iint_{-\infty}^{\infty} d \mathbf{r} d \mathbf{r}^{\prime}\left\langle\delta I^{2}\left(\mathbf{r}, \mathbf{r}^{\prime}\right)\right\rangle \exp \left[i\left(\left(\mathbf{k}_{i}+\mathbf{k}_{f}\right) \pm\left(\mathbf{k}_{i}^{\prime}+\mathbf{k}_{f}^{\prime}\right)\right) \cdot\left(\mathbf{r}-\mathbf{r}^{\prime}\right)\right]
$$

In Eqs. (7) and (8), $A_{l m}$ represents the complex amplitude from the initial scattering point at $\mathbf{r}_{l}$ to the terminal scattering point at $\mathbf{r}_{m}$ [cf. Eqs. (2)-(8) of Ref. 25]. On the other hand, these two points are represented by $r$ and $\mathbf{r}^{\prime}$ in Eq. (9).

It should be observed that for $\mathbf{k}$ vectors out of an angular interval of width $\lambda / W$, centered at $\mathbf{k}_{i}-\mathbf{k}_{i}^{\prime}=\mathbf{k}_{f}-\mathbf{k}_{f}^{\prime}$, the scattered field coherence factor $\mu$ is zero; it should be remarked in this connection that the memory effect is then zero, and hence the $I_{0}$ factor of Eq. (4) becomes one. [We briefly recall that the memory effect, which represents a deterministic relationship between the amplitudes and phases of the incident wave and those of the scattered wave, thus being manifested by a correlation between the resulting speckle patterns as the incident wave vector varies, is given by the square modulus of $\mu$ (Refs. 21 and 23).]

In the limit $n \rightarrow \infty$ the JPDF corresponding to second-order Gaussian statistics [cf., e.g., Eq. (2.94) of Ref. 28] is retrieved:

$$
P_{n}\left(I, I^{\prime}\right)=\frac{\exp (-s)}{\langle I\rangle^{2}\left(1-|\mu|^{2}\right)} I_{0}\left(\frac{2 \sqrt{I I^{\prime}}|\mu|}{\langle I\rangle\left(1-|\mu|^{2}\right)}\right] \text {. }
$$

Due to the aforementioned dependence of the index $n$ on $W$ and on the degree of coherence given by Eq. (9), this limit is obtained either by increasing the size of the scatterer, or by choosing the $k$ vectors out of the ELRC line shape, namely, in directions where the angular degree of coherence $v$ vanishes. Observe that, for both choices of this limit, Eq. (10) holds in particular for angles inside the memory effect line shape of the angular intensity correlation, namely, for $\mathbf{k}$ vectors in proximity to those satisfying $\mathbf{k}_{i}-\mathbf{k}_{i}^{\prime}=\mathbf{k}_{f}-\mathbf{k}_{f}^{\prime}$. Our numerical calculations confirm these statements.

In Figs. 2 and 3 we show the fitting of two sections of the JPDF with the distribution of Eq. (4) for $n=2$ and $n=3$ in the cases $\theta_{0}=3^{\circ}$ and $\theta_{0}=5^{\circ}$. As seen, there is good agreement for low values of the arguments, although at intermediate values of these intensities, the 
fitting is better for the sections of Fig. 2 than for those of the example chosen in Fig. 3.

In summary, we have shown that there exists an intermediate regime in the size of illuminated samples of a random medium, such that the first-order statistics of the reflected wave speckle pattern are Gaussian, but the second-order statistics are not. This occurs at angles where there is interference between time-reversed paths,

${ }^{1}$ Y. Kuga and A. Ishimaru, J. Opt. Soc. Am. A 1, 832 (1984); L. Tsang and A. Ishimaru, ibid. 1, 836 (1984).

${ }^{2}$ M. P. van Albada and A. Lagendijk, Phys. Rev. Lett. 55, 2692 (1985).

${ }^{3}$ P. E. Wolf and G. Maret, Phys. Rev. Lett. 55, 2696 (1985).

${ }^{4}$ E. Akkermans, P. E. Wolf, and R. Maynard, Phys. Rev. Lett. 56, 1471 (1986); E. Akkermans, P. E. Wolf, R. Maynard, and G. Maret, J. Phys. (Paris) 49, 77 (1988).

${ }^{5}$ M. Kaveh, M. Rosenbluth, M. Edrei, and I. Freund, Phys. Rev. Lett. 57, 2049 (1986).

${ }^{6} \mathrm{~S}$. Etemand, R. Thompson, and A. Andrejco, Phys. Rev. Lett. 57, 575 (1986).

${ }^{7}$ V. Celli, A. A. Maradudin, A. M. Marvin, and A. R. McGurn, J. Opt. Soc. Am. 2, 2225 (1985).

${ }^{8}$ K. A. O'Donnell and E. R. Mendez, J. Opt. Soc. Am. A 4, 1194 (1987).

${ }^{9}$ M. Nieto-Vesperinas and J. M. Soto-Crespo, Opt. Lett. 12, 979 (1987); J. M. Soto-Crespo and M. Nieto-Vesperinas, J. Opt. Soc. Am. A 6, 367 (1989).

${ }^{10}$ A. A. Maradudin, E. R. Mendez, and T. Michel, Opt. Lett. 14, 151 (1989); A. A. Maradudin, T. Michel, A. R. McGurn, and E. R. Mendez, Ann. Phys. (N.Y.) 203, 255 (1990).

${ }^{11}$ Scattering and Localization of Classical Waves in Random Media, edited by P. Sheng (World Scientific, Singapore, 1990).

${ }^{12}$ Analogies in Optics and Microelectronics, edited by W. Van Haeringen and D. Lenstra (Kluwer Academic, Dordrecht, and it is in accordance with the existence of the ELRC peaks. Our proposed $K$-joint probability distributions agree well with the numerical results.

This work was supported by the CICYT under Grant No. PB0278. J.A.S-G. acknowledges a grant from the MEC.

The Netherlands, 1990).

${ }^{13}$ Scattering in Volumes and Surfaces, edited by M. NietoVesperinas and J. C. Dainty (North-Holland, Amsterdam, 1990).

${ }^{14}$ N. Garcia and A. Genack, Phys. Rev. Lett. 63, 1678 (1989).

${ }^{15}$ N. Shnerb and M. Kaveh, Phys. Rev. B 43, 1279 (1991).

${ }^{16}$ E. Jakeman and P. N. Pusey, J. Phys. A 8, 369 (1975).

${ }^{17}$ E. Jakeman and R. J. A. Tough, Adv. Phys. 37, 471 (1988).

${ }^{18}$ B. Shapiro, Phys. Rev. Lett. 57, 2168 (1986).

${ }^{19}$ M. Stephen and G. Cwilich, Phys. Rev. Lett. 59, 285 (1987).

${ }^{20}$ A. Z. Genack, Phys. Rev. Lett. 5, 2043 (1987).

${ }^{21}$ S. Feng, C. Kane, P. A. Lee, and D. Stone, Phys. Rev. Lett. 61, 834 (1988).

${ }^{22}$ J. F. de Boer, M. P. van Albada, and A. Lagendijk, Phys. Rev. B 45, 658 (1992).

${ }^{23}$ R. Berkovits and M. Kaveh, Phys. Rev. B 41, 2635 (1990).

${ }^{24}$ R. Berkovits, Phys. Rev. B 42, 10750 (1990).

${ }^{25}$ M. Nieto-Vesperinas and J. A. Sanchez-Gil, Phys. Rev. B 46, 3112 (1992); J. Opt. Soc. Am. A 10, 150 (1993).

${ }^{26}$ J. A. Sanchez-Gil and M. Nieto-Vesperinas, Phys. Rev. B 45, 8623 (1992).

${ }^{27}$ E. Jakeman and P. N. Pusey, IEEE Trans. Antennas Propag. AP-24, 806 (1976).

${ }^{28}$ J. W. Goodman, in Laser Speckle and Related Phenomena, edited by J. C. Dainty (Springer-Verlag, Berlin, 1984), Chap. 2. 